NASA/TM-2003-212027

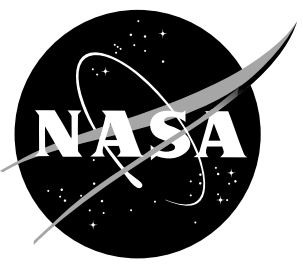

\title{
Real-Time Stability and Control Derivative Extraction From F-15 Flight Data
}

Mark S. Smith and Timothy R. Moes

NASA Dryden Flight Research Center

Edwards, California

Eugene A. Morelli

NASA Langley Research Center

Hampton, Virginia 


\section{The NASA STI Program Office...in Profile}

Since its founding, NASA has been dedicated to the advancement of aeronautics and space science. The NASA Scientific and Technical Information (STI) Program Office plays a key part in helping NASA maintain this important role.

The NASA STI Program Office is operated by Langley Research Center, the lead center for NASA's scientific and technical information. The NASA STI Program Office provides access to the NASA STI Database, the largest collection of aeronautical and space science STI in the world. The Program Office is also NASA's institutional mechanism for disseminating the results of its research and development activities. These results are published by NASA in the NASA STI Report Series, which includes the following report types:

- TECHNICAL PUBLICATION. Reports of completed research or a major significant phase of research that present the results of NASA programs and include extensive data or theoretical analysis. Includes compilations of significant scientific and technical data and information deemed to be of continuing reference value. NASA's counterpart of peer-reviewed formal professional papers but has less stringent limitations on manuscript length and extent of graphic presentations.

- $\quad$ TECHNICAL MEMORANDUM. Scientific and technical findings that are preliminary or of specialized interest, e.g., quick release reports, working papers, and bibliographies that contain minimal annotation. Does not contain extensive analysis.

- CONTRACTOR REPORT. Scientific and technical findings by NASA-sponsored contractors and grantees.
- CONFERENCE PUBLICATION.

Collected papers from scientific and technical conferences, symposia, seminars, or other meetings sponsored or cosponsored by NASA.

- SPECIAL PUBLICATION. Scientific, technical, or historical information from NASA programs, projects, and mission, often concerned with subjects having substantial public interest.

- TECHNICAL TRANSLATION. Englishlanguage translations of foreign scientific and technical material pertinent to NASA's mission.

Specialized services that complement the STI Program Office's diverse offerings include creating custom thesauri, building customized databases, organizing and publishing research results...even providing videos.

For more information about the NASA STI Program Office, see the following:

- Access the NASA STI Program Home Page at http://www.sti.nasa.gov

- E-mail your question via the Internet to help@sti.nasa.gov

- Fax your question to the NASA Access Help Desk at (301) 621-0134

- Telephone the NASA Access Help Desk at (301) 621-0390

- Write to:

NASA Access Help Desk

NASA Center for AeroSpace Information 7121 Standard Drive

Hanover, MD 21076-1320 
NASA/TM-2003-212027

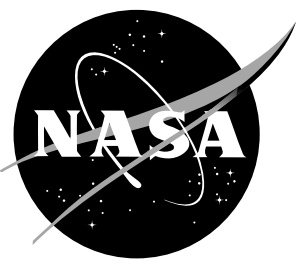

\section{Real-Time Stability and Control Derivative Extraction From F-15 Flight Data}

Mark S. Smith and Timothy R. Moes

NASA Dryden Flight Research Center

Edwards, California

Eugene A. Morelli

NASA Langley Research Center

Hampton, Virginia

National Aeronautics and

Space Administration

Dryden Flight Research Center

Edwards, California 93523-0273 


\section{NOTICE}

Use of trade names or names of manufacturers in this document does not constitute an official endorsement of such products or manufacturers, either expressed or implied, by the National Aeronautics and Space Administration.

Available from the following:

NASA Center for AeroSpace Information (CASI)

7121 Standard Drive

Hanover, MD 21076-1320

(301) 621-0390
National Technical Information Service (NTIS) 5285 Port Royal Road Springfield, VA 22161-2171

(703) $487-4650$ 


\begin{abstract}
A real-time, frequency-domain, equation-error parameter identification (PID) technique was used to estimate stability and control derivatives from flight data. This technique is being studied to support adaptive control system concepts currently being developed by NASA (National Aeronautics and Space Administration), academia, and industry. This report describes the basic real-time algorithm used for this study and implementation issues for onboard usage as part of an indirect-adaptive control system. A confidence measures system for automated evaluation of PID results is discussed. Results calculated using flight data from a modified F-15 aircraft are presented. Test maneuvers included pilot-input doublets and automated inputs at several flight conditions. Estimated derivatives are compared to aerodynamic model predictions. Data indicate that the real-time PID used for this study performs well enough to be used for onboard parameter estimation. For suitable test inputs, the parameter estimates converged rapidly to sufficient levels of accuracy. The devised confidence measures used were moderately successful.
\end{abstract}

\title{
NOMENCLATURE
}

$a_{Y} \quad$ lateral acceleration (positive toward the right), g's

$a_{Z} \quad$ vertical acceleration (positive down), g's

b wingspan, $42.7 \mathrm{ft}$

$\bar{c} \quad$ mean aerodynamic chord, $15.94 \mathrm{ft}$

c.g. aircraft center of gravity

$C_{l} \quad$ rolling moment coefficient

$C_{m} \quad$ pitching moment coefficient

$C_{n} \quad$ yawing moment coefficient

$C_{Y} \quad \mathrm{y}$-axis force coefficient

$C_{Z} \quad$ z-axis force coefficient

DFT discrete Fourier transform

$f \quad$ frequency

FTR Fourier Transform Regression

$g \quad$ acceleration of gravity, $32.174 \mathrm{ft} / \mathrm{s}^{2}$

$\mathrm{Hp} \quad$ pressure altitude, $\mathrm{ft}$

IFCS Intelligent Flight Control System

$I_{x} \quad$ roll moment of inertia, slug- $\mathrm{ft}^{2}$

$I_{x z} \quad$ product of inertia, slug- $\mathrm{ft}^{2}$

$I_{y} \quad$ pitch moment of inertia, slug- $\mathrm{ft}^{2}$

$I_{z} \quad$ yaw moment of inertia, slug- $\mathrm{ft}^{2}$

j imaginary number, $\sqrt{-1}$ 


\begin{tabular}{|c|c|}
\hline$J$ & cost function \\
\hline M & Mach number \\
\hline$m$ & aircraft mass, slugs \\
\hline NASA & National Aeronautics and Space Administration \\
\hline$p$ & roll rate, $\mathrm{rad} / \mathrm{sec}$ \\
\hline PID & parameter identification \\
\hline PreSISE & Prescribed Simultaneous Independent Surface Excitations \\
\hline PTNN & pre-trained neural network \\
\hline$q$ & pitch rate, $\mathrm{rad} / \mathrm{sec}$ \\
\hline $\bar{q}$ & dynamic pressure, psf \\
\hline$r$ & yaw rate, $\mathrm{rad} / \mathrm{sec}$ \\
\hline $\operatorname{Re}$ & real part \\
\hline RFT & recursive Fourier transform \\
\hline$S$ & wing area, $608 \mathrm{ft}^{2}$ \\
\hline$t$ & time \\
\hline$V$ & true airspeed, $\mathrm{ft} / \mathrm{s}$ \\
\hline$X$ & least-squares regressor matrix \\
\hline$Y$ & calculated force or moment coefficient \\
\hline$\alpha$ & angle of attack, deg \\
\hline$\beta$ & angle of sideslip, deg \\
\hline$\delta a$ & aileron deflection, deg \\
\hline Sail & individual aileron deflection, deg \\
\hline$\delta c$ & symmetric canard deflection, deg \\
\hline 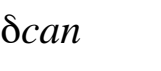 & individual canard deflection, deg \\
\hline$\delta d c$ & differential canard deflection, deg \\
\hline$\delta d t$ & differential stabilator deflection, deg \\
\hline$\delta e$ & elevator (symmetric stabilator) deflection, deg \\
\hline$\delta r$ & rudder deflection, deg \\
\hline \&rud & individual rudder deflection, deg \\
\hline$\delta s$ & individual stabilator deflection, deg \\
\hline$\delta \xi$ & generic control surface deflection, deg \\
\hline$\varepsilon$ & complex equation error \\
\hline$\theta$ & vector of unknown parameters \\
\hline
\end{tabular}


standard error

$\omega$

angular frequency, $\mathrm{rad} / \mathrm{sec}$

Superscripts

$\begin{array}{ll}T & \text { transpose } \\ \sim & \text { estimate } \\ \uparrow & \text { discrete Fourier transform } \\ -1 & \text { complex conjugate transpose } \\ \cdot & \text { matrix inverse } \\ + & \text { time derivative } \\ & \text { tuned-down coefficient }\end{array}$

\section{INTRODUCTION}

Intelligent Flight Control System (IFCS) concepts are currently being developed by NASA (National Aeronautics and Space Administration), academia, and industry. The goal of the IFCS project is to be able to modify in real time the control laws for an aircraft that has been damaged in flight as a result of battle, weather, or a system failure. To perform this reconfiguration, changes in aircraft flight characteristics must in some way be recognized onboard.

The IFCS concept studied here uses aircraft stability and control derivatives to calculate control law gains during flight. ${ }^{1}$ Figure 1 illustrates this concept. ${ }^{1}$ Near-real-time estimation of the stability and control derivatives is required to identify differences from a reference model. These differences are passed to an online-learning neural network, which can learn and recall them based on the flight condition. The recalled derivative differences are added to the reference model and the results are used to generate the new control laws. This IFCS concept is referred to as an indirect-adaptive system.

The process for determining the stability and control derivatives is known as parameter identification (PID) or parameter estimation. With regard to aircraft, this involves analyzing measured aircraft responses from inputs such as control surface movements. PID techniques are generally grouped into three categories: output error, ${ }^{2,3}$ filter error, and equation error. ${ }^{4,5,6}$ Output and filter-error methods are used to solve the nonlinear aircraft equations of motion and are typically used in post-flight analysis. The equation-error techniques are used with system models that are linear in the aerodynamic model parameters, but can otherwise have arbitrary nonlinearities.

The technique used for real-time PID in this study was an equation-error technique known as Fourier transform regression (FTR), which operates in the frequency domain. Flight data were run through the real-time algorithm postflight to evaluate this technique. The low computational requirements of FTR are believed to make it suitable for onboard use. Comparison data were from a linearized model obtained from finite differences using a nonlinear simulation based on wind tunnel data. This paper presents results from flight studies and details the effectiveness of using the real-time algorithm for stability and control derivative identification. The paper also discusses some of the implementation issues that must be addressed to enable future online real-time parameter identification using the FTR technique. 


\section{AIRPLANE DESCRIPTION}

Figure 2 shows the airplane used for this research. The airplane is a pre-production Boeing (McDonnell Aircraft Company, now the Boeing Company, Chicago, Illinois) F-15B that has been highly modified to support various test programs. The most visible modifications are canards added near the pilot station that are modified horizontal stabilators from the Boeing F-18 aircraft. The propulsion system consists of two Pratt \& Whitney (West Palm Beach, Florida) F100-PW-229 engines, each equipped with an axisymmetric thrust vectoring pitch-yaw balance beam nozzle. The thrust-vectoring feature was disabled for the flights described in this report. The reference areas and lengths used for nondimensionalizing forces and moments are listed in Table 1.

Table 1. Test aircraft reference dimensions.

\begin{tabular}{lc}
\hline \hline Wing area & $608 \mathrm{ft}^{2}$ \\
Mean aerodynamic chord & $15.94 \mathrm{ft}$ \\
Wing span & $42.7 \mathrm{ft}$ \\
Moment reference & FS 557.2, WL 116.3, BL 0.0 \\
\hline \hline
\end{tabular}

\section{Control Surfaces}

The test airplane has five pairs of control surfaces: canards, ailerons, trailing-edge flaps, stabilators, and rudders. Flaps and aileron droop are manually set by the pilot and only used for takeoff and landing configurations. Conventional pitch control is provided by symmetric deflection of the all-moving horizontal stabilators and canards. Roll control uses aileron and differential stabilator, which is sometimes referred to as differential tail deflection. Directional control is provided by rudder deflection and differential canard. Definitions of control surface deflections are as follows:

Pitch effectors:

$$
\begin{gathered}
\delta e=0.5\left(\delta s_{\text {left }}+\delta s_{\text {right }}\right) \\
\delta c=0.5\left(\delta \text { can }_{\text {left }}+\delta \text { can }_{\text {right }}\right)
\end{gathered}
$$

Yaw-roll effectors:

$$
\begin{aligned}
& \delta r=0.5\left(\delta r_{u d} d_{l e f t}+\delta r u d_{\text {right }}\right) \\
& \delta a=\delta a i l_{l e f t}-\delta a i l_{\text {right }} \\
& \delta d t=\delta s_{\text {left }}-\delta s_{\text {right }} \\
& \delta d c=\delta c a n_{l e f t}-\delta c a n_{\text {right }}
\end{aligned}
$$




\section{Instrumentation and Data Acquisition}

The test airplane was equipped with a complete set of air data, inertial, and control surface position instrumentation. Input data were provided to the PID algorithm at $40 \mathrm{~Hz}$. All parameters used in this study are from analog measurement sources, except Mach number, true airspeed, pressure altitude, roll angle, and pitch angle. These parameters are from the Military Standard 1553 Databus, as they would be for an onboard system. Time latencies in these parameters would not have a substantial effect on the PID results.

Indicated angles of attack and sideslip from the noseboom were corrected for boom offset angles, noseboom bending, angular rates, upwash and sidewash. Measured vertical and lateral accelerations were corrected to the center of gravity. All state and control variables were filtered to remove biases by using low-pass filters with very low cutoffs to identify steady-state components that were then subtracted from the original signals. All of these data processing calculations were performed as part of the real-time PID system.

Accurate estimates of weight, center of gravity (c.g.), and inertias were required for each flight maneuver. Fuel weight measurements were obtained from three fuselage and two wing tank sensors. A model was used to calculate mass properties estimates based on the fuel weights at each data point. This model was included in the data processing portion of the PID algorithm.

\section{MANEUVER DESCRIPTION}

This section describes the two types of flight test maneuvers analyzed in this study. Examples of control-surface time histories for each kind are presented.

\section{Pilot Inputs}

The primary flight test maneuvers used in this study were pilot inputs. In particular, square doublets were used because of their desirable frequency content. ${ }^{3}$ Doublets were typically separated into two categories: pitch and yaw-roll. The yaw and roll doublets were combined to separate out the effects of coupling in the lateral-directional responses. Pilot inputs are advantageous because they can be performed quickly and throughout the entire flight envelope.

Figure 3 shows that pilot stick and rudder inputs result in high correlation between the symmetric canard and angle of attack, rudder and differential canard, and differential stabilator and aileron. This correlation renders the control effectiveness derivatives unidentifiable because the PID technique cannot discern which surface is causing the response. This problem can be alleviated by altering the PID technique, as will be discussed later in this paper, and by using automated surface inputs.

\section{Automated Surface Excitations}

Programmed test inputs were also used for this study. These were automated stacked sine wave inputs, which simultaneously moved all control surfaces at unique frequencies. These maneuvers, called Prescribed Simultaneous Independent Surface Excitations (PreSISE), were hard coded into the flight computer. The duration of the inputs was fifteen seconds. PreSISE maneuvers alleviate the 
aforementioned correlation problems and provide a means of estimating all stability and control parameters at the same time. The control surface deflections are calculated using the equation:

$$
\delta \xi=A\left(\sin \left(f_{1} t\right)+\sin \left(f_{2} t\right)\right)
$$

where $A$ is the amplitude, which was selected by the pilot. Three amplitudes were available: $0.2,0.4$, and 0.8 degrees. The parameters $f_{1}$ and $f_{2}$ determined the frequency characteristics of the inputs, varied for each collective control surface, and are shown in Table 2. Figure 4 shows an example of a 0.8 deg PreSISE maneuver. These maneuvers are added to the commanded surface positions, so the overall positions will be influenced by trim values, control system feedback, and pilot inputs during the maneuver.

Table 2. Frequencies used for PreSISE maneuvers.

\begin{tabular}{lcc}
\hline \hline \multicolumn{1}{c}{ Surface } & $f_{1}(\mathrm{~Hz})$ & $f_{2}(\mathrm{~Hz})$ \\
\hline$\delta e$ & 0.3125 & 0.9375 \\
$\delta c$ & 0.6250 & 1.2500 \\
$\delta d t$ & 0.6250 & 1.5625 \\
$\delta a$ & 1.2500 & 2.1875 \\
$\delta r$ & 0.9375 & 0.0000 \\
$\delta d c$ & 0.3125 & 1.8750 \\
\hline \hline
\end{tabular}

\section{METHODS OF ANALYSIS}

This section describes the analytical technique used for this study. The basic real-time PID algorithm will be discussed along with modifications that were made to it.

\section{PID Technique}

The real-time PID method used for this study is FTR, developed by Morelli. ${ }^{5}$ Equation-error PID assumes that the individual force and moment coefficients of the airplane can be expressed as sums of component terms as follows:

$$
\begin{gathered}
C_{Z}=C_{Z_{o}}+C_{Z_{\alpha}} \alpha+C_{Z_{\delta e}} \delta e+C_{Z_{\delta c}} \delta c \\
C_{Y}=C_{Y_{0}}+C_{Y_{\beta}} \beta+C_{Y_{\delta \alpha}} \delta \alpha+C_{Y_{\delta r}} \delta r+C_{Y_{\delta d c}} \delta d c+C_{Y_{\delta d t} \delta d t} \\
C_{m}=C_{m_{0}}+C_{m_{\alpha}} \alpha+C_{m_{q}} \frac{q \bar{c}}{2 V}+C_{m_{\delta e}} \delta e+C_{m_{\delta c}} \delta c \\
C_{l}=C_{l_{0}}+C_{l_{\beta}} \beta+C_{l_{p}} \frac{p b}{2 V}+C_{l_{r}} \frac{r b}{2 V}+C_{l_{\delta a}} \delta a+C_{l_{\delta r}} \delta r+C_{l_{\delta d c}} \delta d c+C_{l_{\delta d t}} \delta d t
\end{gathered}
$$




$$
C_{n}=C_{n_{0}}+C_{n_{\beta}} \beta+C_{n_{p}} \frac{p b}{2 V}+C_{n_{r}} \frac{r b}{2 V}+C_{n_{\delta a}} \delta a+C_{n_{\delta r}} \delta r+C_{n_{\delta} d c} \delta d c+C_{n_{\delta} d t} \delta d t
$$

The roll and yaw rates, $p$ and $r$, are nondimensionalized using the wingspan, $b$, and the velocity, $V$. Pitch rate, $q$, is nondimensionalized using the reference chord, $\bar{c}$, and velocity. Terms that multiply the stability and control derivatives are called regressors. The number and type of regressors will often vary depending on whether the force or moment coefficient is longitudinal or lateral-directional. Typically, the force equations will not include rate derivatives. For this study, no bias terms (such as $C_{m_{0}}$ ) were estimated for any of the force and moment coefficients.

The coefficient expansions of equations (1) through (5) can be rewritten in the form

$$
Y(t)=X(t) \theta
$$

where $Y$ is the force or moment coefficient, $X$ is the matrix of regressors, and $\theta$ is the matrix of unknowns. For the pitching moment case,

$$
\begin{gathered}
Y=C_{m} \\
X=\left[\begin{array}{lll}
1 & \alpha \frac{q \bar{c}}{2 V} \delta e \delta c
\end{array}\right] \\
\theta=\left[\begin{array}{l}
C_{m_{0}} \\
C_{m_{\alpha}} \\
C_{m_{q}} \\
C_{m_{\delta e}} \\
C_{m_{\delta c}}
\end{array}\right]
\end{gathered}
$$

The objective of parameter estimation is to solve equation (6) for the unknown coefficients in $\theta$.

The left sides of equations (1) through (5) are the force and moment coefficients that are computed using measured flight data. The force coefficients are computed using the measured accelerations, after they are translated to the c.g. location, and the following equations:

$$
\begin{aligned}
& C_{Z}=\frac{m g}{\bar{q} S} a_{Z} \\
& C_{Y}=\frac{m g}{\bar{q} S} a_{Y}
\end{aligned}
$$


Axial force derivatives were not considered in this study, because they do not exert much influence on important flying qualities and they typically require good thrust measurements.

The moment coefficients are computed using inertias, angular rates, and angular accelerations. ${ }^{3}$ For a symmetric aircraft,

$$
\begin{gathered}
C_{l}=\left[\dot{p} I_{x}-\dot{r} I_{x z}-q r\left(I_{y}-I_{z}\right)-p q I_{x z}\right] / \bar{q} S b \\
C_{m}=\left[\dot{q} I_{y}-\left(r^{2}-p^{2}\right) I_{x z}-r p\left(I_{z}-I_{x}\right)\right] / \bar{q} S \bar{c} \\
C_{n}=\left[-\dot{p} I_{x z}+\dot{r} I_{z}-p q\left(I_{x}-I_{y}\right)+q r I_{x z}\right] / \bar{q} S b
\end{gathered}
$$

The use of angular accelerations in these equations poses problems for real-time parameter estimation and is one of the reasons why frequency domain techniques are attractive. Angular accelerations are typically computed by numerical differentiation, which can lead to signal noise and time skew problems. In the frequency domain, time derivatives are easily computed algebraically.

After the force and moment coefficients are computed, a least-squares regression technique can be used to estimate the derivatives. For frequency domain regression, the data are put into the frequency domain via a Fourier transform. Real-time frequency domain methods use recursive Fourier transforms.

The finite Fourier transform of the signal $x(t)$ is defined by 5

$$
\tilde{x}(\omega) \equiv \int_{0}^{T} x(t) e^{-j \omega t} d t
$$

which can be approximated using the equation

$$
\tilde{x}(\omega) \approx \Delta t \sum_{i=0}^{N-1} x_{i} e^{-j \omega t_{i}}
$$

This can be rewritten as

$$
\tilde{x}(\omega) \approx X(\omega) \Delta t
$$

where $\Delta t$ is the time step and $X(\omega)$ is the discrete Fourier transform (DFT). The DFT of a parameter $x(t)$ at time step $i$ can be expressed as: ${ }^{5}$

$$
X_{i}(\omega)=X_{i-1}(\omega)+x_{i} e^{-j \omega i \Delta t}
$$

where $j=\sqrt{-1}$ and

$$
e^{-j \omega i \Delta t}=e^{-j \omega \Delta t} e^{-j \omega(i-1) \Delta t}
$$


Equations (18) and (19) show that the Fourier transform at any time step can be expressed as a function of the data at that time step and the Fourier transform at the previous time step, hence the name recursive Fourier transform (RFT). The simplicity of the RFT calculation makes this technique very advantageous for real-time applications.

The force and moment coefficients and regressors of equations (1) through (5) were transformed into the frequency domain using this technique. Equation (6) can then be formulated as a standard least-squares regression problem with complex data, ${ }^{5}$

$$
\tilde{Y}=\tilde{X} \theta+\varepsilon
$$

where

$$
\begin{gathered}
\tilde{Y}=\left[\begin{array}{c}
\tilde{y}\left(\omega_{1}\right) \\
\tilde{y}\left(\omega_{2}\right) \\
\cdot \\
\cdot \\
\cdot \\
\tilde{y}\left(\omega_{n}\right)
\end{array}\right] \\
\tilde{X}=\left[\begin{array}{c}
\tilde{x}\left(\omega_{1}\right) \\
\tilde{x}\left(\omega_{2}\right) \\
\cdot \\
\cdot \\
\cdot \\
\tilde{x}\left(\omega_{n}\right)
\end{array}\right]
\end{gathered}
$$

and $\varepsilon$ represents the complex equation error in the frequency domain. The parameter $n$ is the number of frequencies used in the DFT. For PID, it is important to obtain the Fourier transforms at frequencies of interest to rigid body flight mechanics. Confining the data analysis to the frequency band where the system dynamics reside automatically filters out wide-band measurement noise and structural response modes that are typically outside of the frequency band of interest. ${ }^{5}$ For the F-15 analysis, thirty-six discrete frequencies were used from $0.1 \mathrm{~Hz}$ to $1.5 \mathrm{~Hz}$ at $0.04 \mathrm{~Hz}$ increments. Increasing the number of frequencies will increase computational time and memory requirements.

The least-squares cost function is:

$$
J=\frac{1}{2}(\tilde{Y}-\tilde{X} \theta)^{\dagger}(\tilde{Y}-\tilde{X} \theta)
$$

The parameter vector estimate that minimizes the cost function is 5 


$$
\hat{\theta}=\left[\operatorname{Re}\left(\tilde{X}^{\dagger} \tilde{X}\right)\right]^{-1} \operatorname{Re}\left(\tilde{X}^{\dagger} \tilde{Y}\right)
$$

and the estimated parameter covariance matrix is ${ }^{5}$

$$
\operatorname{cov}(\hat{\theta})=\frac{1}{(n-p)}|\tilde{Y}-\tilde{X} \theta|^{2}\left[\operatorname{Re}\left(\tilde{X}^{\dagger} \tilde{X}\right)\right]^{-1}
$$

where $p$ in this case is the number of elements in the vector $\hat{\theta}$. The parameter standard errors, $\sigma$, are calculated by taking the square root of the diagonal of the covariance matrix.

\section{Data Forgetting}

For flight purposes, the running sum of the RFT may produce problems because it will contain information from data at older flight conditions that might be vastly different from the current ones. Since stability and control parameters vary with flight condition, this would cause the least-squares problem to be corrupted. As such, the real-time PID algorithm was modified to compute parameter estimates from a specified time window by removing old data from the RFT. With this modification, the DFT shown in equation (18) becomes:

$$
X_{i}(\omega)=X_{i-1}(\omega)+x_{i} e^{-j \omega i \Delta t}-x_{i-k} e^{-j \omega(i-k) \Delta t}
$$

for $i>k$ and $k$ is the number of data points in the window,

$$
k=\frac{t_{\text {window }}}{\Delta t}
$$

The size of the window should be chosen to balance information quantity and sensitivity to flight condition. The addition of data forgetting cancels one of the advantages of using the RFT, because some time history of data must be retained. This adds complexity to the system and increases memory requirements.

\section{Dealing With Correlated Inputs}

As mentioned before, pilot inputs cause many of the signals used in the PID analysis to be correlated for this particular test airplane. While the automated PreSISE maneuvers should alleviate these problems at discrete test points, a practical real-time application would require that the PID algorithm work sufficiently with only pilot inputs. Therefore, changes were made to the algorithm in an attempt to remove the correlation problems. The resulting system was referred to as tuned-down.

In the tuned-down version of the FTR method, half of the stability and control derivatives were removed from the estimation problem. Specifically, the aileron, canard, and angular rate derivatives were eliminated. Reference values were used as substitutes for derivatives that were not estimated. These values came from a nonlearning neural network, referred to as the pre-trained neural network (PTNN). The PTNN served as a table lookup based on Mach number, $\alpha, \beta$, altitude, and control surface positions. 
The contributions of these terms were removed from the total forces and moments, creating new coefficients:

$$
\begin{aligned}
& C_{Z}^{\prime}=C_{Z}-C_{Z_{\delta c, P T N N}} \delta c \\
& C_{m}^{\prime}=C_{m}-C_{m_{\delta c, P T N N}} \delta c-C_{m_{q, P T N N}} \frac{q \bar{c}}{2 V} \\
& C_{Y}^{\prime}=C_{Y}-C_{Y_{\delta d c, P T N N}} \delta d c-C_{Y_{\delta a, P T N N}} \delta a \\
& C_{l}^{\prime}=C_{l}-C_{l_{\delta d c, P T N N}} \delta d c-C_{l_{\delta a, P T N N}} \delta a-\left(C_{l_{p, P T N N}} \frac{p b}{2 V}-C_{l_{r, P T N N}} \frac{r b}{2 V}\right) \\
& C_{n}{ }^{\prime}=C_{n}-C_{n_{\delta d c, P T N N}} \delta d c-C_{n_{\delta a, P T N N}} \delta a-\left(C_{n_{p, P T N N} 2 V} \frac{p b}{2 V} C_{n_{r, P T N N}} \frac{r b}{2 V}\right)
\end{aligned}
$$

where the primed terms are the new coefficients to be used in the regression. These computations were done before the data were transformed into the frequency domain.

While eliminating correlation issues, tuning down the FTR method in this way introduces some accuracy issues. Any errors in the fixed parameters will result in bias errors in the estimated parameters.

\section{Confidence Measures}

For incorporation of the real-time PID technique into an onboard adaptive system, it is important that the system has a way of knowing whether or not the parameter estimates are acceptable. Accomplishing this with a single criterion is difficult. A system of confidence tests can provide better results. The goal is a system that filters out all inaccurate estimates, but still allows sufficient amounts of good data to pass through.

One of the primary tools available for checking the validity of the PID results are the parameter standard errors, $\sigma$, which are computed as previously mentioned. The standard errors indicate mathematical confidence in the estimated derivatives. One way to evaluate the quality of the PID results is to check the computed standard errors versus limits. If the errors are below the limits, then the PID estimates will be deemed acceptable. Standard errors can also be used to calculate relative errors, which are the standard errors divided by the absolute value of the parameter estimates. These errors can be checked against defined limits that are specified for each parameter. Relative errors are a way of evaluating the sizes of the standard errors in relation to the sizes of the derivatives. This is a potential problem because it tightens the accuracy requirement as the absolute value of the estimated parameter shrinks.

Simulation studies indicated that using error-based tests alone might not be sufficient for differentiating good results from bad, especially during situations in which inputs to the system were small or nonexistent. To solve this problem, another test was developed that checks the information 
content in the frequency-domain force and moment coefficient data. Parseval's Theorem can be used to obtain the equation for the power contained in the data for each coefficient: ${ }^{7}$

$$
\int_{-\infty}^{\infty}|\tilde{Y}(\omega)|^{2} d \omega
$$

This integration can be done numerically over the frequency range of the analysis and the resulting value can be compared to specified bounds to determine if the information content is sufficient to get acceptable PID results. If there is too little information, the parameter estimates will automatically be deemed unacceptable. If sufficient information is present, overall confidence will still depend on the results of the error-related tests. This method alone can exclude many inaccurate derivative estimates.

For this study, a confidence measure system has been designed using a combination of these tests. Figure 5 illustrates this system, which also includes a persistence test that was added to block out sporadic points that might slip through the other tests. The standard error test is used to overlay the persistence test results. If both are passed, the estimated stability or control derivative will be considered good. The confidence measure system was designed to provide flexibility and a means of evaluating the usefulness of each individual test.

Figure 6 shows a schematic of the persistence test. Results of the information content and relative error tests are passed to the persistence test, which uses a counter to determine the validity of the results. This counter is limited to the range of zero to five and is incremented by one whenever the information and relative errors tests are both passed. When the value is at three or higher, the persistence test is passed. From a zero counter, estimates must pass the relative error and information tests three straight times before passing the persistence test, which acts as a way of specifying a convergence time. When at least one of those tests has been failed, the counter is reduced by three. The result is that, after a string of good values, a single failed point does not reset the counter completely. The persistence test was designed to allow a considerable amount of control over its behavior.

Bounds for the information content and error tests were determined using simulation results. Noise was added to the simulation data prior to doing PID to better reflect flight data. PreSISE maneuvers and doublets were simulated at several flight conditions. The FTR results were analyzed to determine when the derivative estimates were converged. The standard and relative error bounds were then specified so that the confidence tests would be passed at the proper times. Since the results vary somewhat with flight condition and maneuver type and amplitude, engineering judgment was used to balance them. Table 3 shows the resulting standard and relative error bounds. The information content bounds shown in Table 4 were developed in the same manner. 
Table 3. Simulation-based error bounds used for this study.

\begin{tabular}{ccc}
\hline \hline Derivative & standard error $(1 / \mathrm{deg})$ & relative error \\
\hline$C_{Z_{\alpha}}$ & $8.35 \mathrm{e}-04$ & 0.10 \\
$C_{Z_{\delta e}}$ & $4.69 \mathrm{e}-04$ & 0.10 \\
$C_{m_{\alpha}}$ & $6.05 \mathrm{e}-04$ & 0.10 \\
$C_{m_{\delta e}}$ & $2.47 \mathrm{e}-04$ & 0.10 \\
$C_{Y_{\beta}}$ & $7.74 \mathrm{e}-04$ & 0.10 \\
$C_{Y_{\delta d t}}$ & $1.37 \mathrm{e}-04$ & 0.10 \\
$C_{Y_{\delta r}}$ & $1.98 \mathrm{e}-04$ & 0.10 \\
$C_{l_{\beta}}$ & $1.79 \mathrm{e}-04$ & 0.10 \\
$C_{l_{\delta d t}}$ & $4.98 \mathrm{e}-05$ & 0.10 \\
$C_{l_{\delta r}}$ & $8.12 \mathrm{e}-05$ & 0.25 \\
$C_{n_{\beta}}$ & $4.79 \mathrm{e}-04$ & 0.15 \\
$C_{n_{\delta d t}}$ & $7.36 \mathrm{e}-05$ & 0.25 \\
$C_{n_{\delta r}}$ & $1.77 \mathrm{e}-04$ & 0.15 \\
\hline
\end{tabular}

Table 4. Simulation-based information content bounds used for this study.

\begin{tabular}{cc}
\hline \hline Coefficient & Limit \\
\hline$C_{Z}$ & $9 \times 10^{-3}$ \\
$C_{Y}$ & $5 \times 10^{-5}$ \\
$C_{m}$ & $9 \times 10^{-5}$ \\
$C_{l}$ & $6 \times 10^{-7}$ \\
$C_{n}$ & $3 \times 10^{-6}$ \\
\hline \hline
\end{tabular}

RESULTS AND DISCUSSION

Flight test maneuvers were performed primarily at three flight conditions: Mach 0.75 and an altitude of 20,000 feet, Mach 0.90 and an altitude of 25,000 feet, and Mach 1.2 and an altitude of 32,000 feet. The PreSISE maneuvers were flown at these conditions only, typically as a set of three of each magnitude. Sets of pitch, roll, and yaw doublets were also done at these conditions, and sequences were flown in which the pilot performed doublets frequently while varying flight conditions. Data from all of these maneuvers were run through the real-time PID algorithm postflight. Some ground testing of the entire adaptive system, with PID and both the learning and pre-trained neural networks, has been done on a research computer that can be installed onboard the airplane. This computer had three $400-\mathrm{MHz}$ 
processors and $128 \mathrm{MB}$ of RAM. The tests indicate that the FTR algorithm can, in fact, be used for near real-time PID in flight.

Comparison data were obtained by linearizing the aerodynamic model used by the nonlinear simulator. Inaccuracies are expected in the aerodynamic model data, by their nature, so they cannot be used to truly judge the accuracy of the PID results. The aerodynamic model, however, is useful for assessing the reasonableness of the parameter estimates.

Parameter estimates were run through the aforementioned confidence measure system. The error bounds derived by simulation data were occasionally much lower than the standard and relative errors in the flight-determined results. While not perfect, the results from the confidence tests help to illustrate which estimates are good and are therefore a reasonable indication of the values that would be passed to a learning neural network in an adaptive control scheme. The results can also be used to refine the error bounds and the confidence measures system in general. Derivative estimates that passed the confidence tests are referred to as valid points and will be shown as symbols in the figures.

\section{Effects of Data Forgetting}

Some consideration must be given to the way in which the use of data forgetting affects the PID results. Figure 7 shows a comparison of estimated $C_{n_{\delta r}}$ with and without the use of data forgetting, along with estimated standard errors. The data retention window was 10 seconds. Symbols indicate the points that passed the confidence tests. Comparison values from the linearized aerodynamic model are shown. Since these results come from just a portion of the flight data, they are not a perfect replication of what would be seen if the PID algorithm had been running for the entire flight, especially in the no-data-forgetting case.

This figure illustrates how FTR with data forgetting converges onto a value as the maneuver is performed and remains there for a period after the maneuver is complete. Ten seconds after the PreSISE maneuver ends, all of the data relating to it have been forgotten and FTR begins to produce bad estimates because it no longer has information with which to work. When the next maneuver is initiated, FTR again begins to calculate good estimates. While both versions of FTR converge to approximately the same value, the results of the data-forgetting version are slightly noisier, and remain so, for the duration of the PreSISE maneuver. Note that for the case without data forgetting, additional maneuvers do not seem to appreciably decrease the standard errors. It is not uncommon for the standard errors to drift slightly up. This indicates that the dynamics are changing slightly, which is expected. This is one way the estimator shows that the model is changing. Also of note is that the standard error plot mirrors the derivative plot, which shows that the standard errors accurately indicate the quality of the parameter estimation results.

For the test airplane, $C_{m_{\alpha}}$ is an ideal parameter to demonstrate the necessity of data forgetting. The presence of canards makes the aircraft statically longitudinally unstable at subsonic speeds. At supersonic speeds, however, the aircraft is stable. As such, $C_{m_{\alpha}}$ changes signs across Mach 1. Figure 8 shows $C_{m_{\alpha}}$ estimated using tuned-down FTR for a maneuver sequence that goes from subsonic to supersonic speeds and back. Also shown are the measured pressure altitude (Hp) and Mach number. The sequence begins subsonically with some PreSISE maneuvers, followed by doublets. After attaining supersonic speed, 
some general (non-PID) maneuvering was done, followed by a single PreSISE maneuver. The aircraft slowed back down to subsonic speeds for a turn, then returned to the supersonic flight condition for two more PreSISE maneuvers. From the figure, it can be seen that FTR identifies the change in $C_{m_{\alpha}}$ when data forgetting is used.

A small data-retention window would be necessary to detect a failure in a period of time suitable for an adaptive control system. It would also be required for cases in which flight conditions are rapidly changing. The window would need to be large enough, however, to retain sufficient information to produce good estimates. For the remainder of this study, a ten second retention window is used. This window length balances the size requirements with an emphasis on the accuracy of the parameter estimates.

\section{Tuned-Down vs. Normal FTR}

Since the onboard algorithm requires tuned-down FTR to analyze piloted doublets, it is necessary to evaluate the differences between the tuned-down results and those of the normal FTR. This is possible by analyzing PreSISE maneuvers, since their uncorrelated nature allows use of the unmodified FTR. Analysis of PID results at the three flight conditions indicates that errors due to tuning down vary by parameter and by flight condition. Figure 9 shows results for $C_{m_{\alpha}}$ at the Mach 0.75 condition. In this case, the tuned-down and normal FTR results do not match. This indicates that errors exist in the assumed PTNN values for the non-estimated derivatives $C_{m_{q}}$ and $C_{m_{\delta c}}$. To test this, the $C_{m_{q}}$ and $C_{m_{\delta c}}$ estimates from the full FTR were used in place of the PTNN values for the tuned-down PID algorithm. As Figure 10 shows, the resulting $C_{m_{\alpha}}$ estimates then matched the full FTR result.

\section{Doublet Results}

Because this study was based on an implementable real-time algorithm for the test airplane, attention is focused on the FTR results for pilot input doublets. A more thorough analysis of PreSISE maneuver results has been presented elsewhere. ${ }^{8}$ Figures 11 through 15 show all FTR results for a doublet sequence at Mach 0.9 and an altitude of 25,000 ft. This sequence consists of three sets of pitch, yaw, and roll doublets. For these results, the tuned-down FTR technique was used with the PTNN derivatives substituting for the non-estimated parameters.

Figure 11 shows the two estimated z-axis force derivatives for the tuned-down FTR, $C_{Z_{\alpha}}$ and $C_{Z_{\delta e}}$, along with the estimated standard errors for the elevator derivative. No points for either derivative passed the confidence tests, though $C_{Z_{\alpha}}$ matches the aerodynamic model well. This indicates that the error bounds for $C_{Z_{\alpha}}$ might have been too restrictive. The figure shows that the standard error for $C_{Z_{\delta e}}$ is much higher than the error bound. The substantial difference between the estimated $C_{Z_{\delta e}}$ and the aerodynamic model value suggest that the FTR results may be inaccurate for this derivative, though it is possible that the aerodynamic model is in error. If the PID results are in error, the confidence tests worked as intended by not declaring any of the derivatives as valid. The nearly flat spots in the derivatives following the maneuvers are present because very little information is being added to the 
data - so the least-squares problem is not changing until information is removed by means of the data-forgetting capability. Changes in the estimated derivatives do appear, however, when subsequent doublets are performed.

The three estimated y-force derivatives are shown in Figure 12. All three derivatives match the aerodynamic model very well. Several points passed the confidence tests, especially for $C_{Y_{\beta}}$. The results for $C_{Y_{\beta}}$ indicate that the information-content test for $C_{Y}$ was passed for most of the sequence, which suggests that the simulation-derived bound might be too permissive. The other two derivatives, $C_{Y_{\delta d t}}$ and $C_{Y_{\delta r}}$, did not pass the confidence tests as often as $C_{Y_{\beta}}$ because they failed the error tests. Note that $C_{Y_{\delta r}}$ converges shortly after the pedal input, whereas $C_{Y_{\delta d t}}$ requires the lateral stick input.

Figure 13 shows the two estimated pitching moment derivatives, $C_{m_{\alpha}}$ and $C_{m_{\delta e}}$, and the estimated standard errors for $C_{m_{\delta e}}$. Both derivatives disagree somewhat with the predicted values from the aerodynamic model. Good repeatability is shown for $C_{m_{\delta e}}$, however, though no points passed the confidence tests. Again, the preset bound on standard error was too low. For $C_{m_{\alpha}}$, results from the first and last doublet agree well. The results for the second doublet, which do not agree, did not pass the confidence tests. Specifically, the relative error test was failed for that portion of the results. The relative error was roughly 20 percent and the boundary was 10 percent.

Results for the rolling moment derivatives are shown in Figure 14. Two of these derivatives, $C_{l_{\beta}}$ and $C_{l_{\delta d t}}$, passed the confidence tests many times. Good repeatability was demonstrated for all three derivatives. Estimates for $C_{l_{\beta}}$ agree well with the aerodynamic model, but estimates of the other two derivatives do not. $C_{l_{\delta r}}$ converged to a different sign than the prediction, but did not pass all of the confidence tests. $C_{l_{\delta r}}$ passed the standard error test, but not the relative error test. It is inconclusive whether the aerodynamic model or the PID results are in error.

Figure 15 shows the results for the yawing moment derivatives. All three estimated parameters $\left(C_{n_{\beta}}\right.$, $C_{n_{\delta r}}$, and $C_{n_{\delta d t}}$ ) demonstrated good repeatability. They also passed the confidence tests very often and agreed well with the aerodynamic model. For the most part, the yawing moment derivatives converged quickly to a solid answer. While convergence time varies by maneuver and is dependent on the input, FTR results typically converged a couple of seconds after the maneuver.

The figures indicate that the confidence measure system worked adequately for this study. In some cases, the simulation-based error bounds were too low, and some derivative estimates that may have been accurate did not pass the tests. Examination of the results also indicates some areas where the confidence tests were not as selective as they should be. This can be seen, for example, in the results for $C_{n_{\delta d t}}$, where a wide range of values passed the tests. Much work would be required to improve the confidence measures, by either tuning the various error bounds, or developing a new system and/or new tests. 


\section{CONCLUDING REMARKS}

Intelligent Flight Control System (IFCS) concepts are currently being developed by NASA, academia, and industry. The goal of the IFCS project is to be able to modify in real time the control laws for an airplane that has been damaged in flight as a result of battle, weather, or a system failure. In some IFCS implementations, near real-time stability and control parameter identification (PID) is necessary for successfully reconfiguring the control system. Initial flight tests have been conducted to gather data to assess the accuracy and robustness of proposed on-board implementations of stability and control PID techniques. This paper presents results of one such technique that uses a frequency-domain, equation-error approach called Fourier transform regression (FTR).

Results generated using flight data have shown that the FTR technique provides accurate stability and control derivative estimates in real time. These results generally compared well with aerodynamic model predictions. The PID algorithm was modified to work around correlation problems that occur with pilot inputs by replacing some of the terms with a priori values, which introduced some error in the results. Results indicate that a data-forgetting capability must be incorporated into the FTR method if continuous operation at changing flight conditions is required.

A confidence measure system was devised to automatically determine accurate stability and control derivative estimates from inaccurate ones. Such a system would be required for an indirect-adaptive control system to tell the learning neural network the data on which to train. The confidence tests were based on standard and relative errors, information content, and persistence. The tests' error and information content bounds were based on simulation studies. Flight results indicated that many of these bounds were too restrictive. The confidence measure system was marginally successful overall. These results can be used to improve the definition of confidence measures for future tests. 


\section{REFERENCES}

1. Hageman, Jacob J., Mark S. Smith, and Susan Stachowiak, Integration of Online Parameter Identification and Neural Network for In-Flight Adaptive Control, AIAA-2003-5700, August 2003.

2. Murray, James E., and Richard E. Maine, pEst Version 2.1 User's Manual, NASA TM 88280, September 1987.

3. Maine, Richard E., and Kenneth W. Iliff, Application of Parameter Estimation to Aircraft Stability and Control: the Output-Error Approach, NASA RP 1168, June 1986.

4. Morelli, Eugene A., Real-Time Parameter Estimation in the Frequency Domain, AIAA-99-4043, 1999.

5. Morelli, Eugene A., In-Flight System Identification, AIAA 98-4261, 1998.

6. Klein, V., Aircraft Parameter Estimation in Frequency Domain, AIAA 78-1344, 1978.

7. Beauchamp, K. G., Signal Processing Using Analog and Digital Techniques, page 337, John Wiley \& Sons, New York, 1973.

8. Moes, Timothy R., Mark S. Smith, and Eugene A. Morelli, Flight Investigation of Prescribed Simultaneous Independent Surface Excitations (PreSISE) for Real-Time Stability and Control Derivative Estimation, AIAA-2003-5702, Atmospheric Flight Mechanics Conference, Austin, TX, August 2003. 


\section{FIGURES}

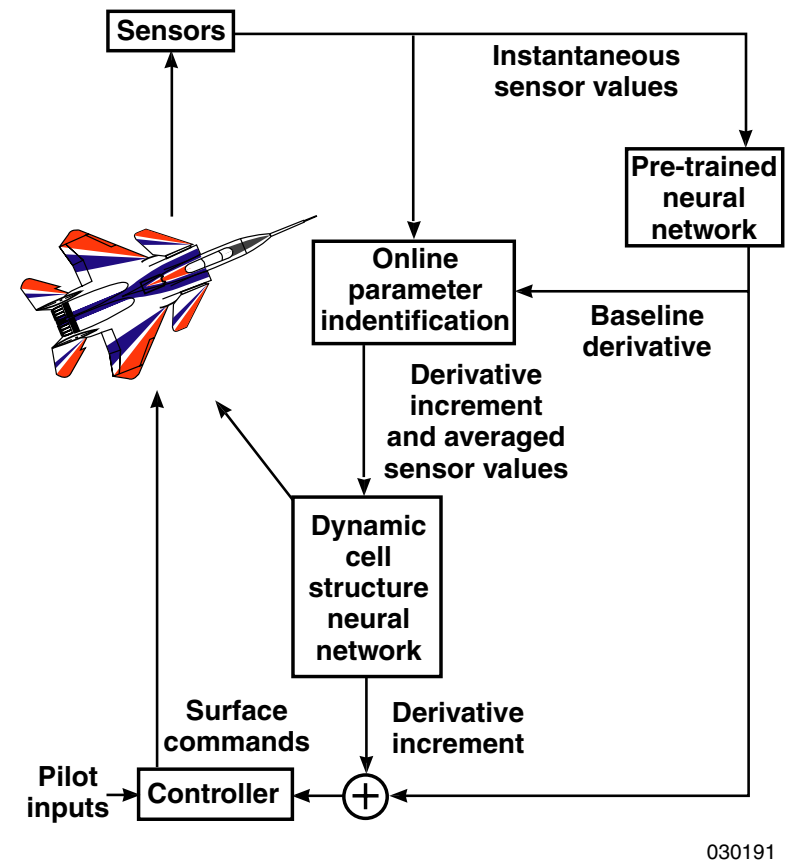

Figure 1. Schematic of IFCS concept.
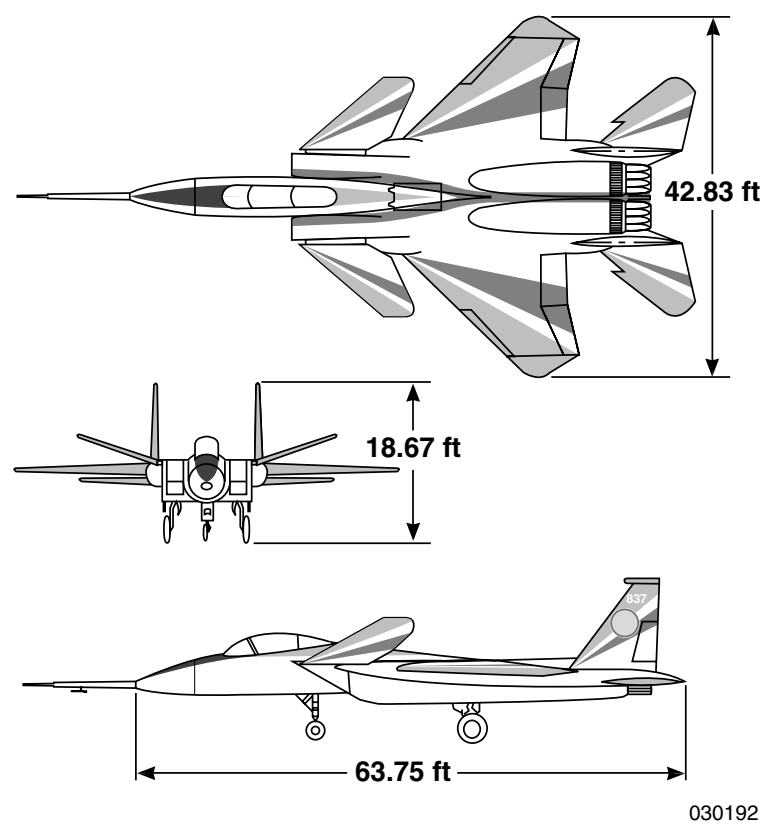

Figure 2. F-15 IFCS aircraft. 

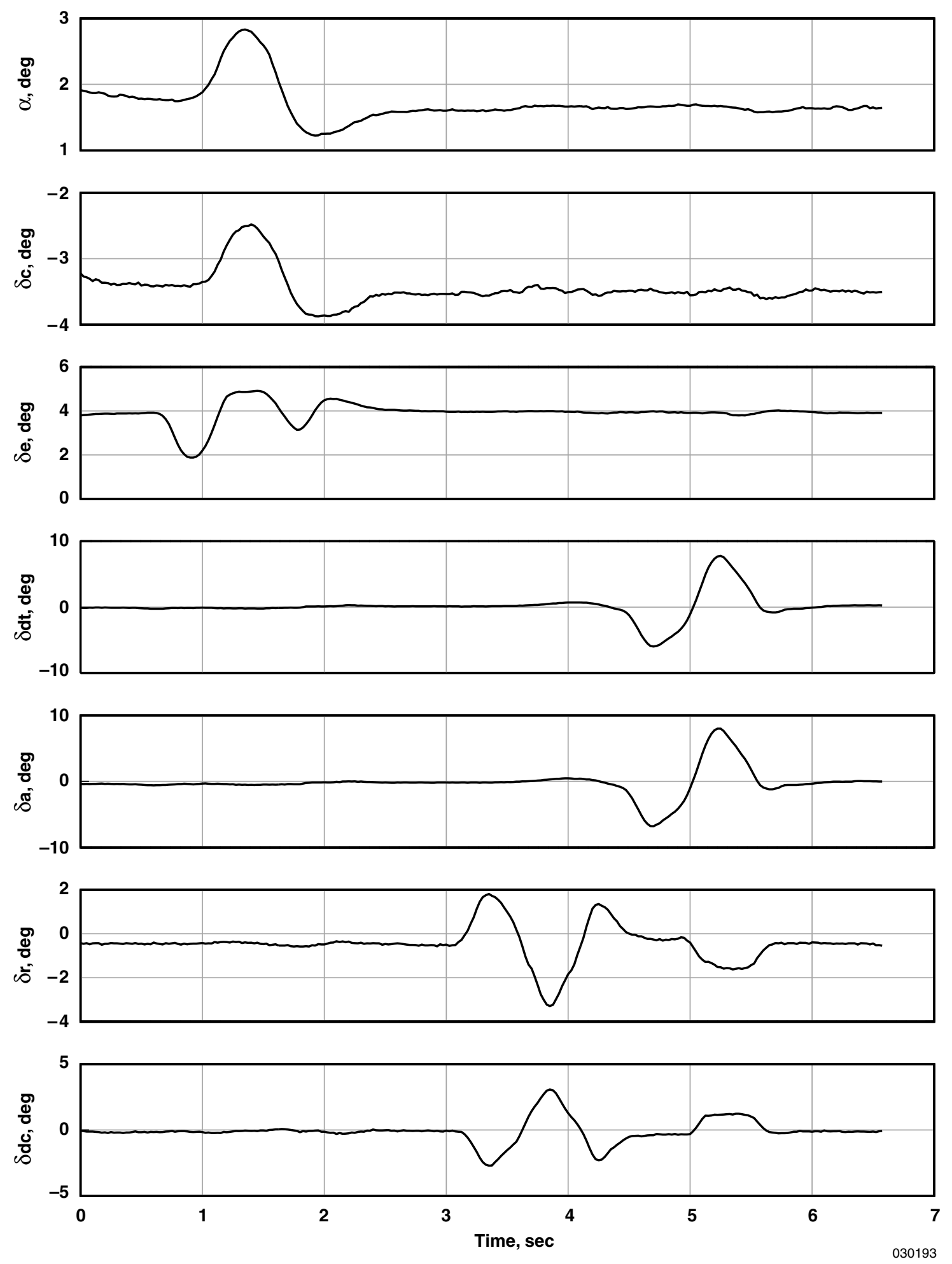

Figure 3. Time history of control surfaces for pilot input doublets. 

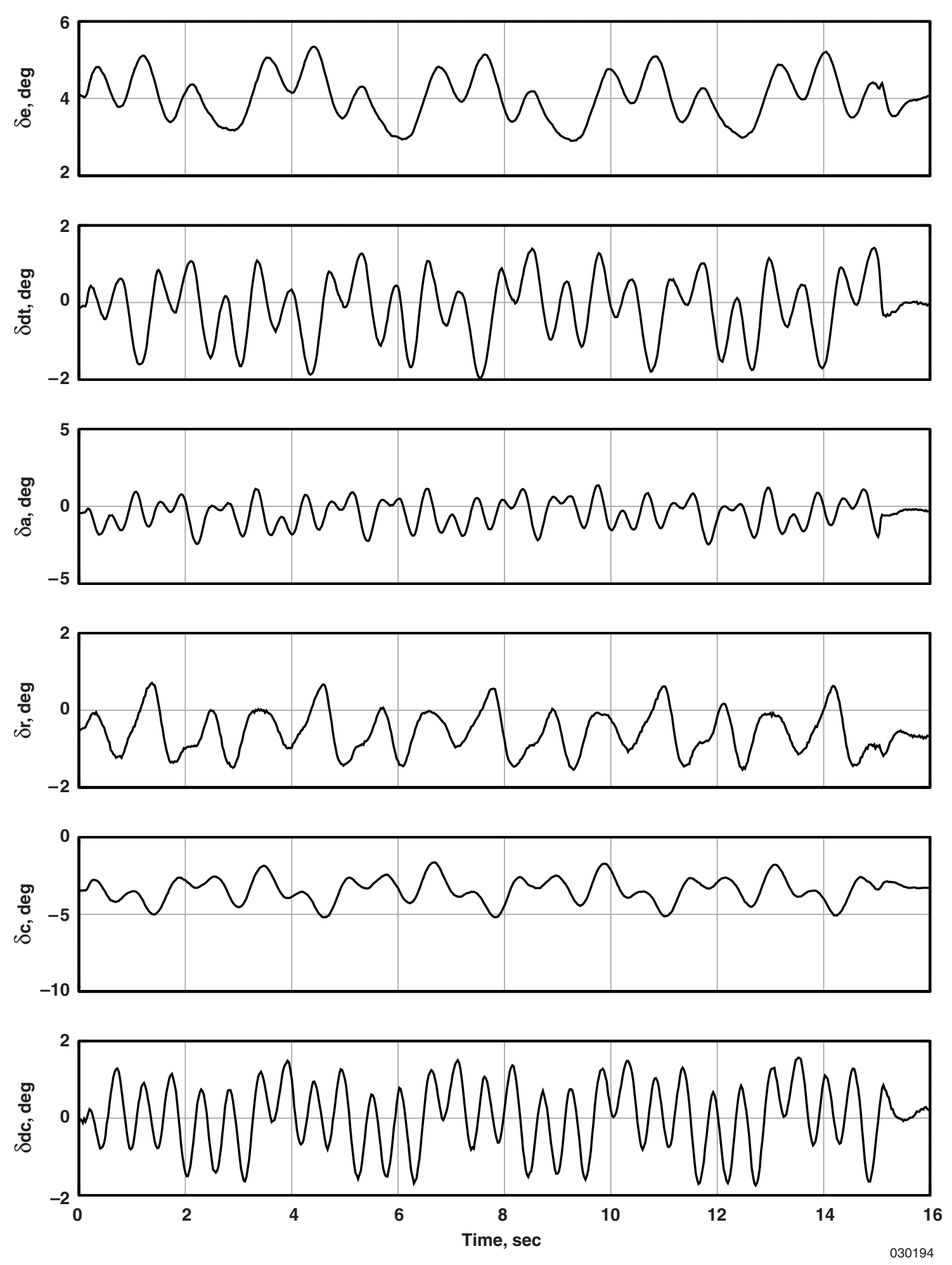

Figure 4. Example of a PreSISE maneuver 


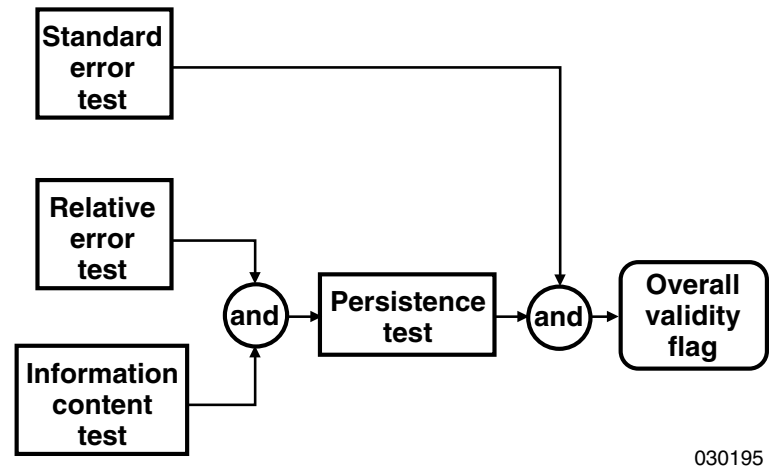

Figure 5. Overview of confidence measures system

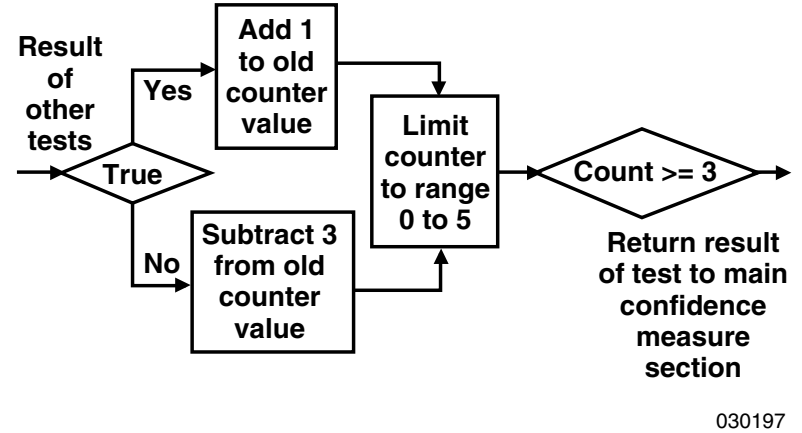

Figure 6. Diagram of consistency / persistence test 


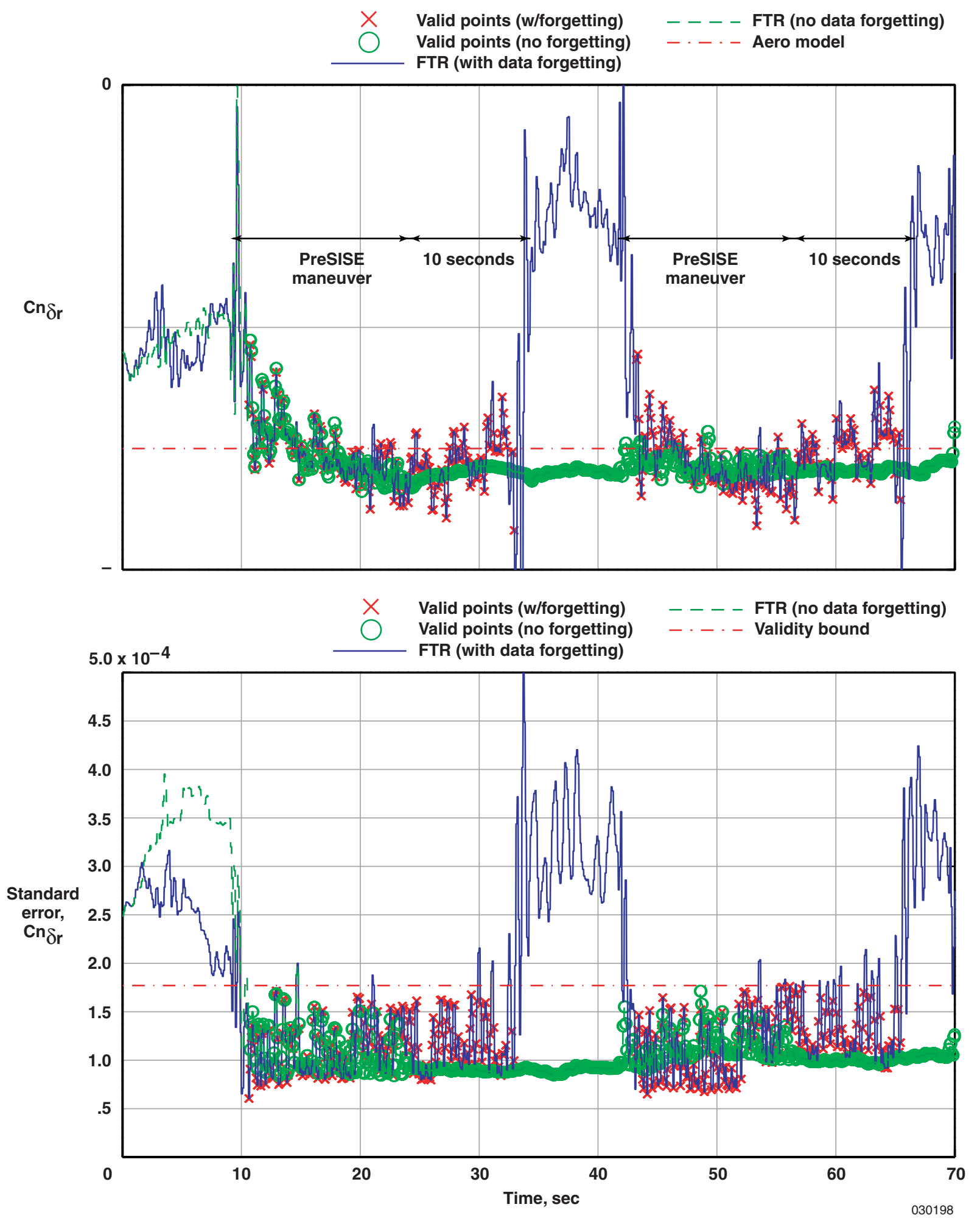

Figure 7. Effect of data forgetting on FTR results $(\mathrm{M}=0.9, \mathrm{Hp}=25,000 \mathrm{ft})$. 

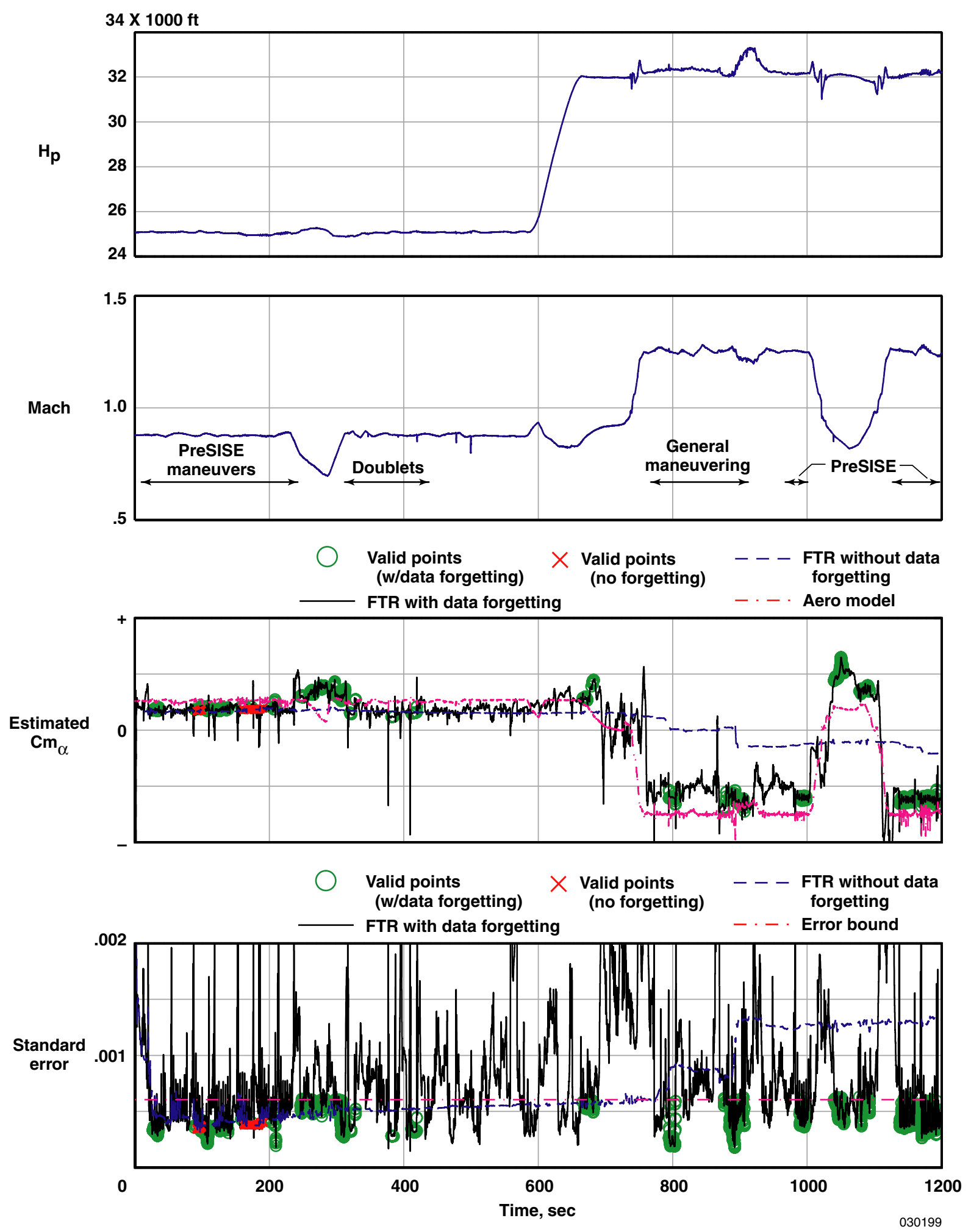

Figure 8. Long-term effects of data forgetting on FTR results. 


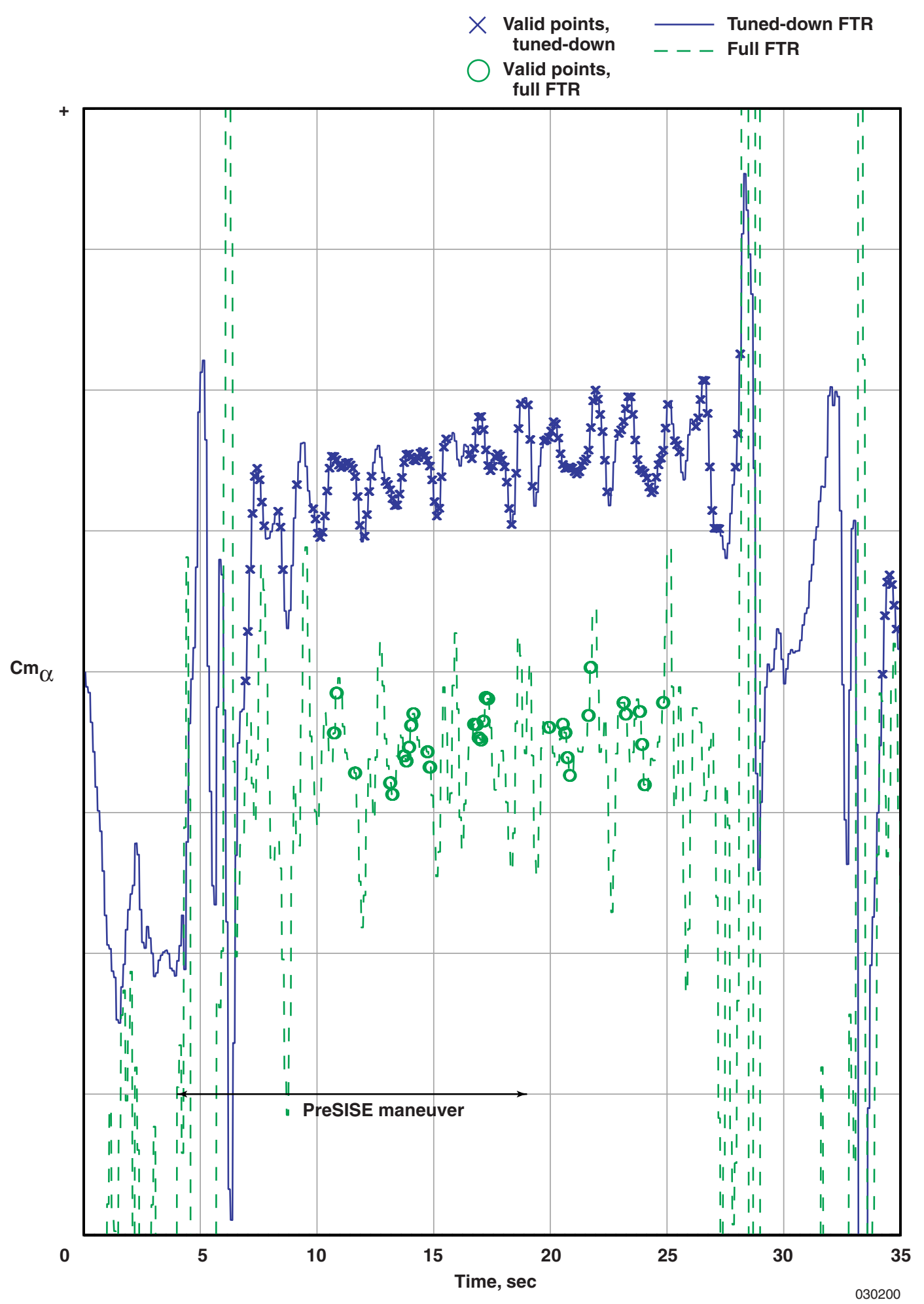

Figure 9. Comparison of tuned-down and full FTR results $(\mathrm{M}=0.75, \mathrm{Hp}=20,000 \mathrm{ft})$. 


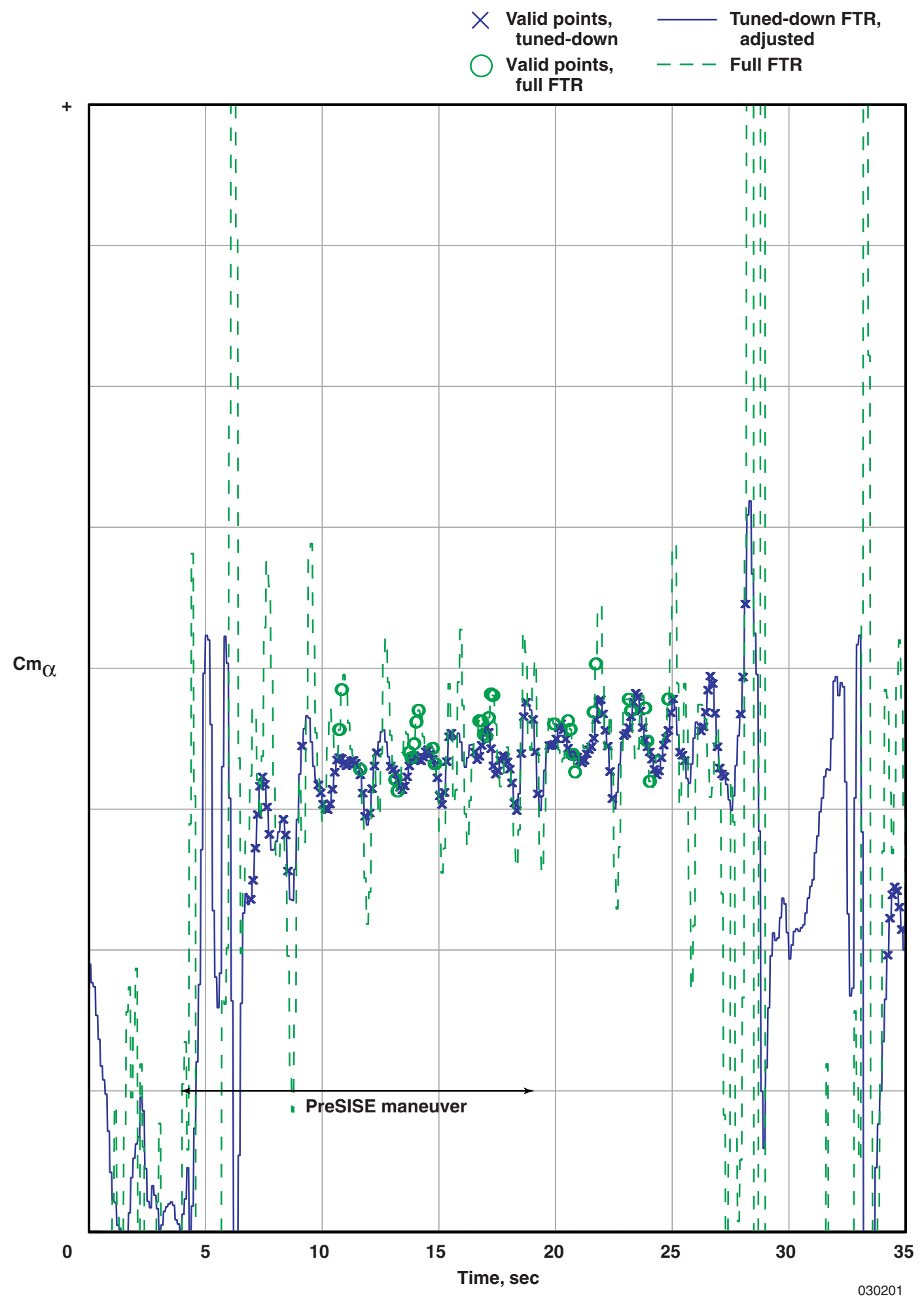

Figure 10. Comparison of full FTR with adjusted tuned-down FTR $(\mathrm{M}=0.75, \mathrm{Hp}=20,000 \mathrm{ft})$. 

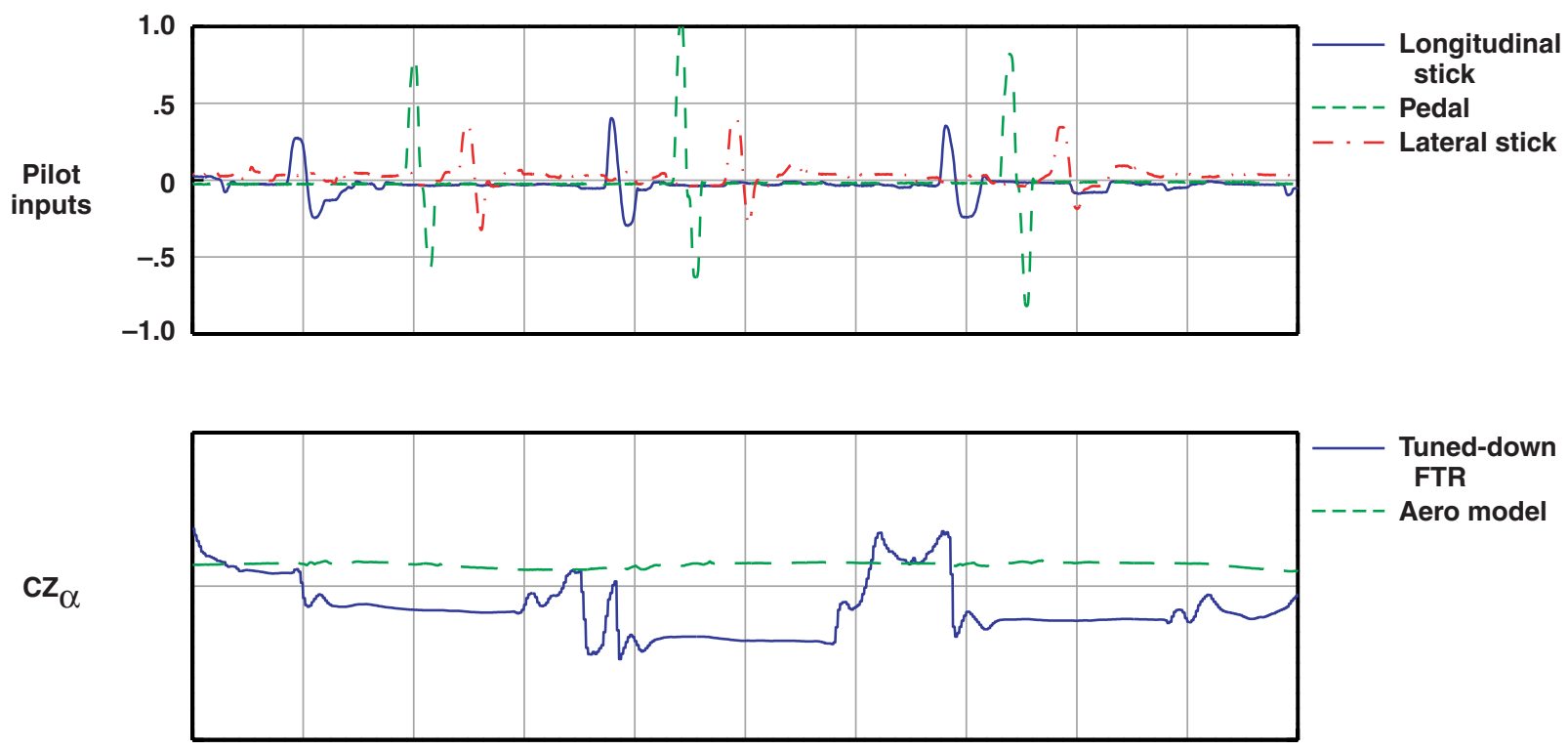

Tuned-down

FTR

---- Aero model
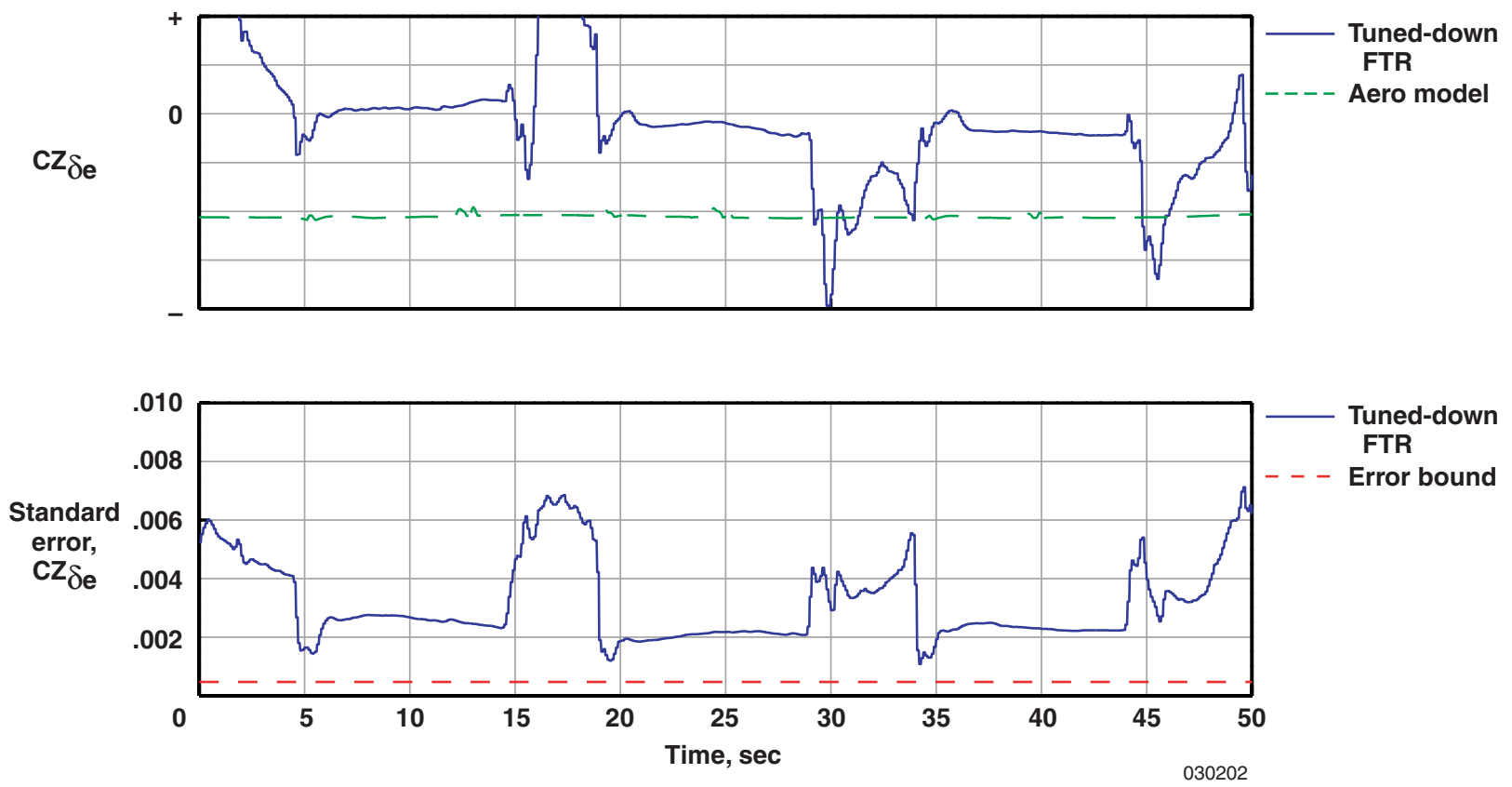

Figure 11. Estimates of $\mathrm{z}$-axis force derivatives $(\mathrm{M}=0.9, \mathrm{Hp}=25,000 \mathrm{ft})$. 

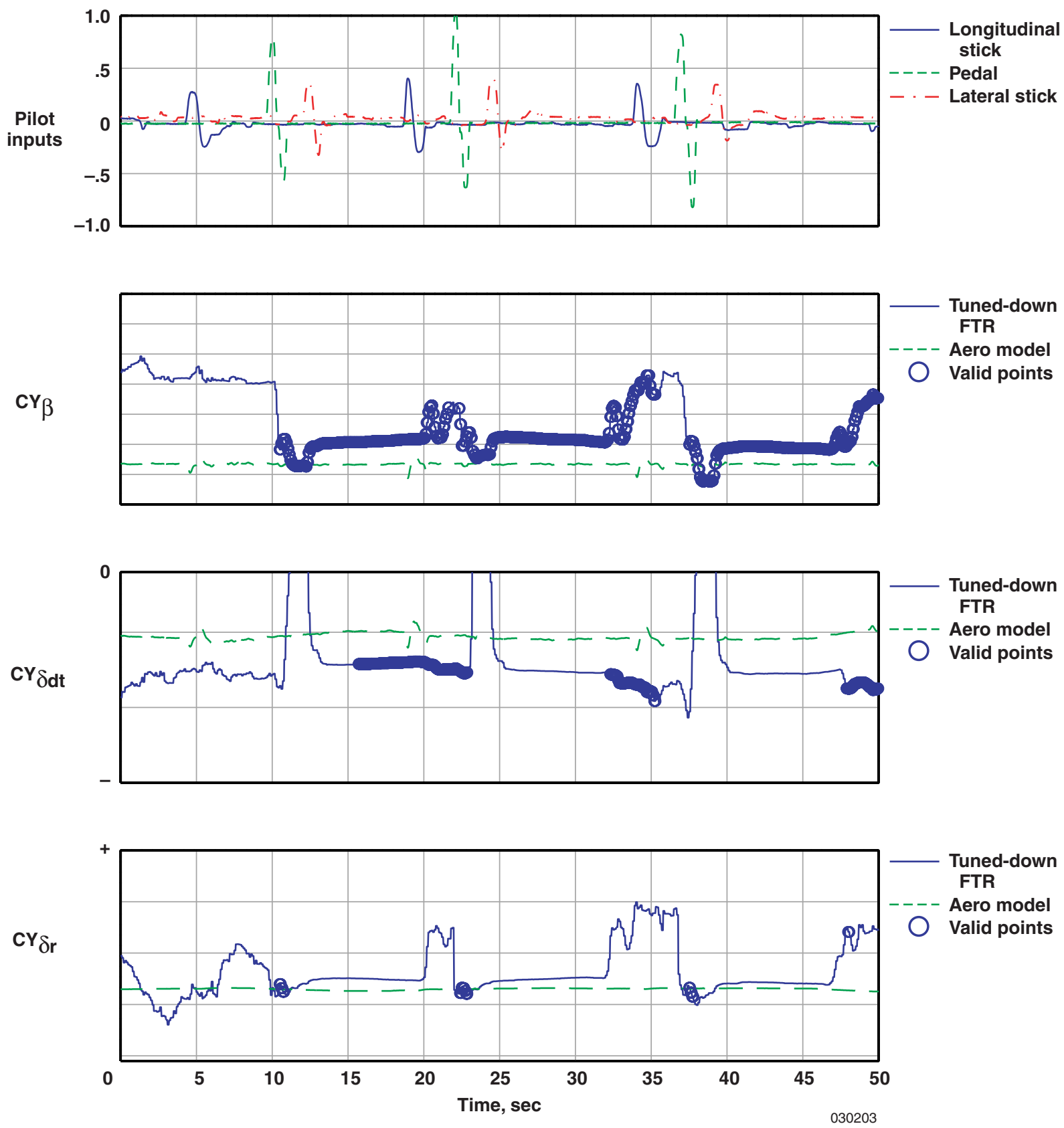

Figure 12. Estimates of $\mathrm{y}$-axis force derivatives $(\mathrm{M}=0.9, \mathrm{Hp}=25,000 \mathrm{ft})$. 

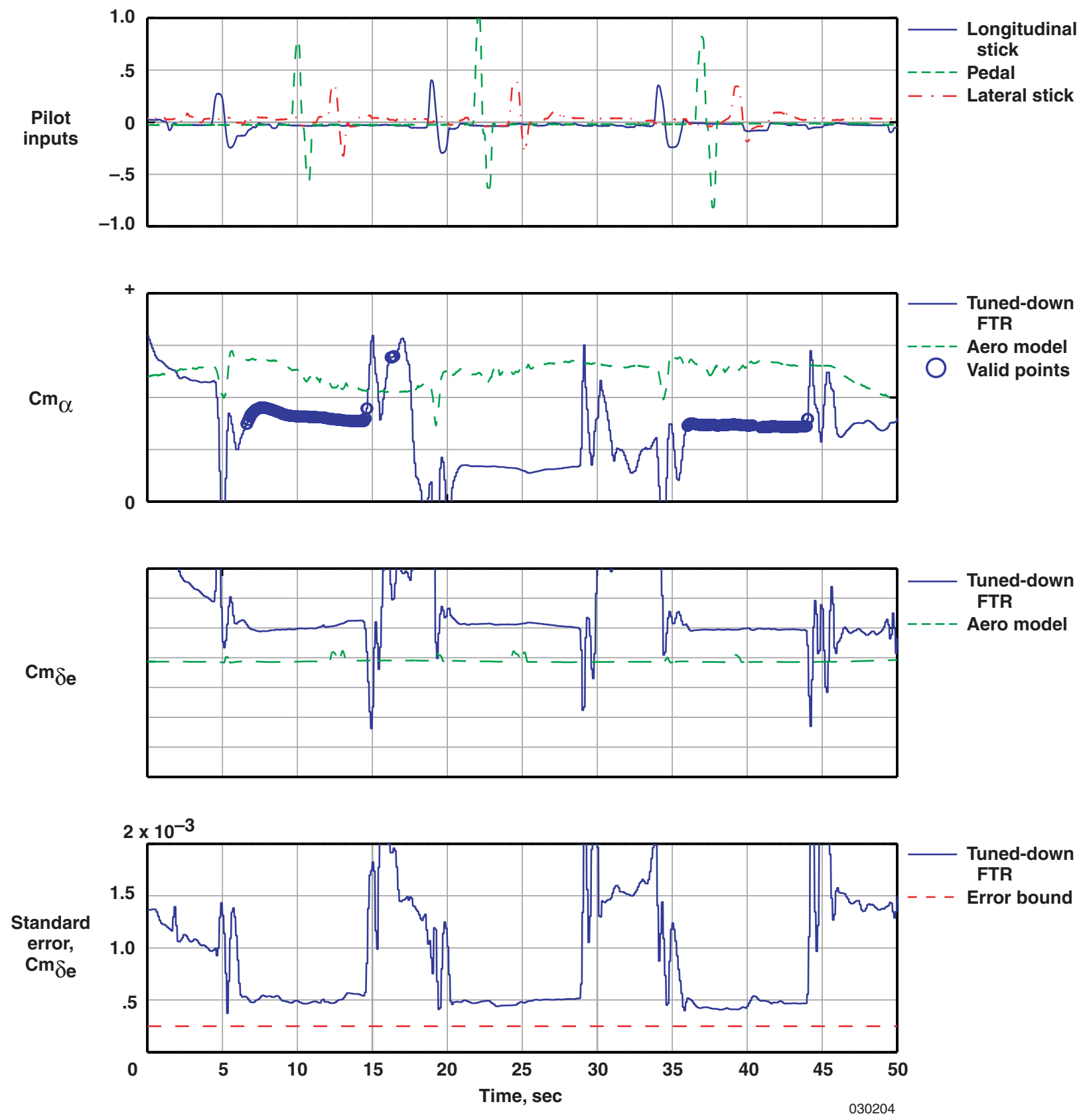

Figure 13. Estimates of pitching moment derivatives $(\mathrm{M}=0.9, \mathrm{Hp}=25,000 \mathrm{ft})$. 

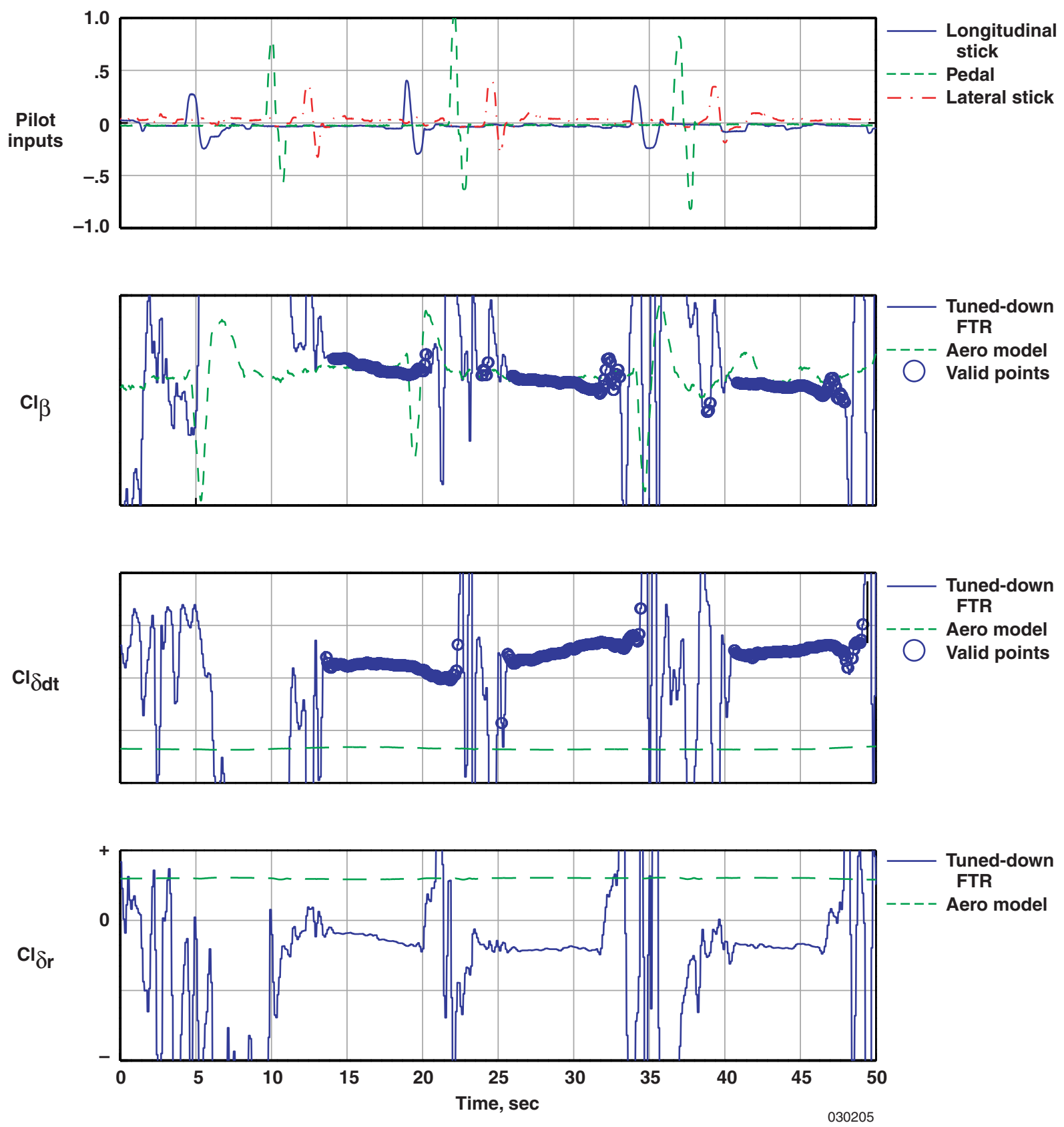

Figure 14. Estimates of rolling moment derivatives $(\mathrm{M}=0.9, \mathrm{Hp}=25,000 \mathrm{ft})$. 

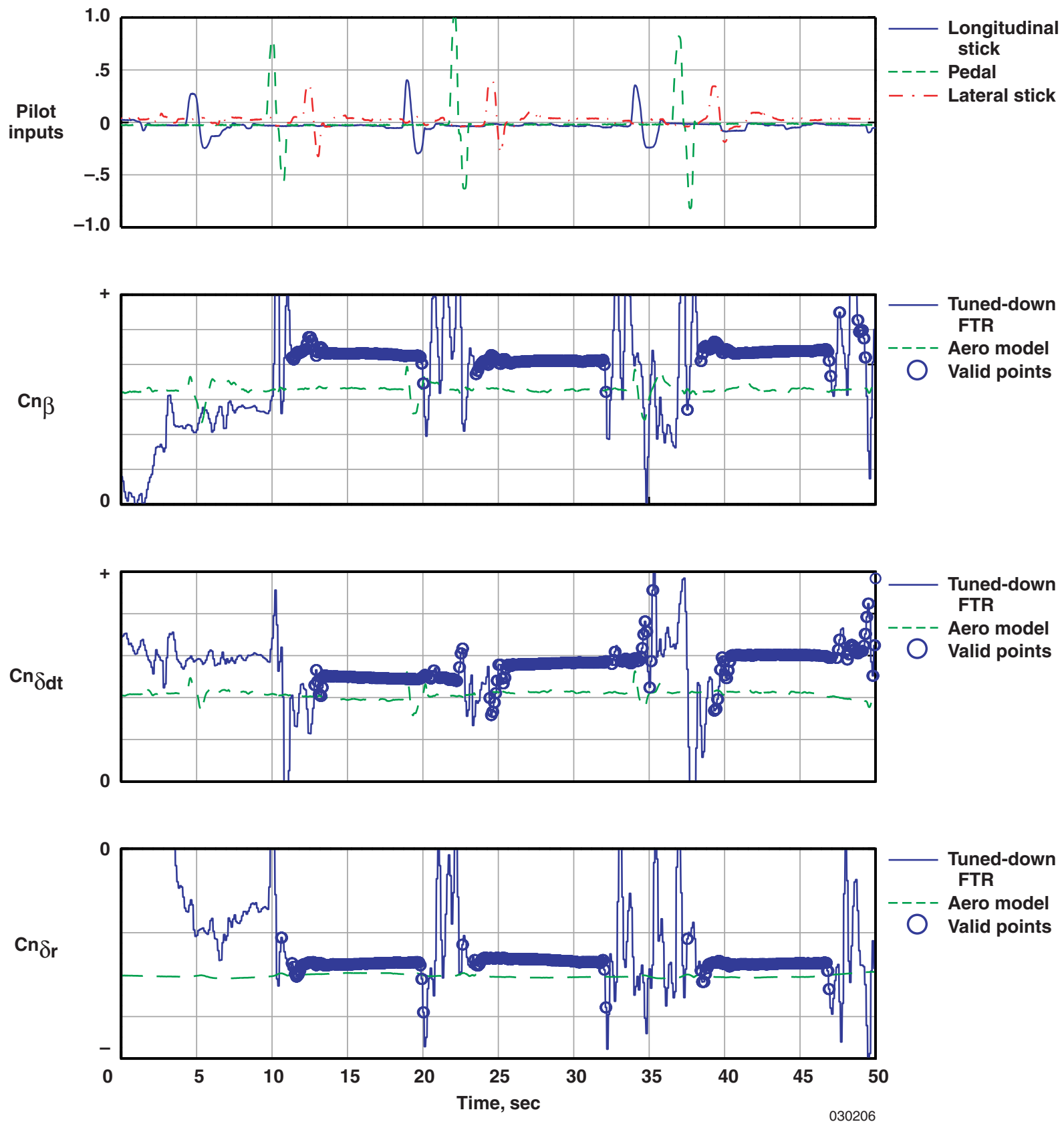

Figure 15. Estimates of yawing moment derivatives $(\mathrm{M}=0.9, \mathrm{Hp}=25,000 \mathrm{ft})$. 


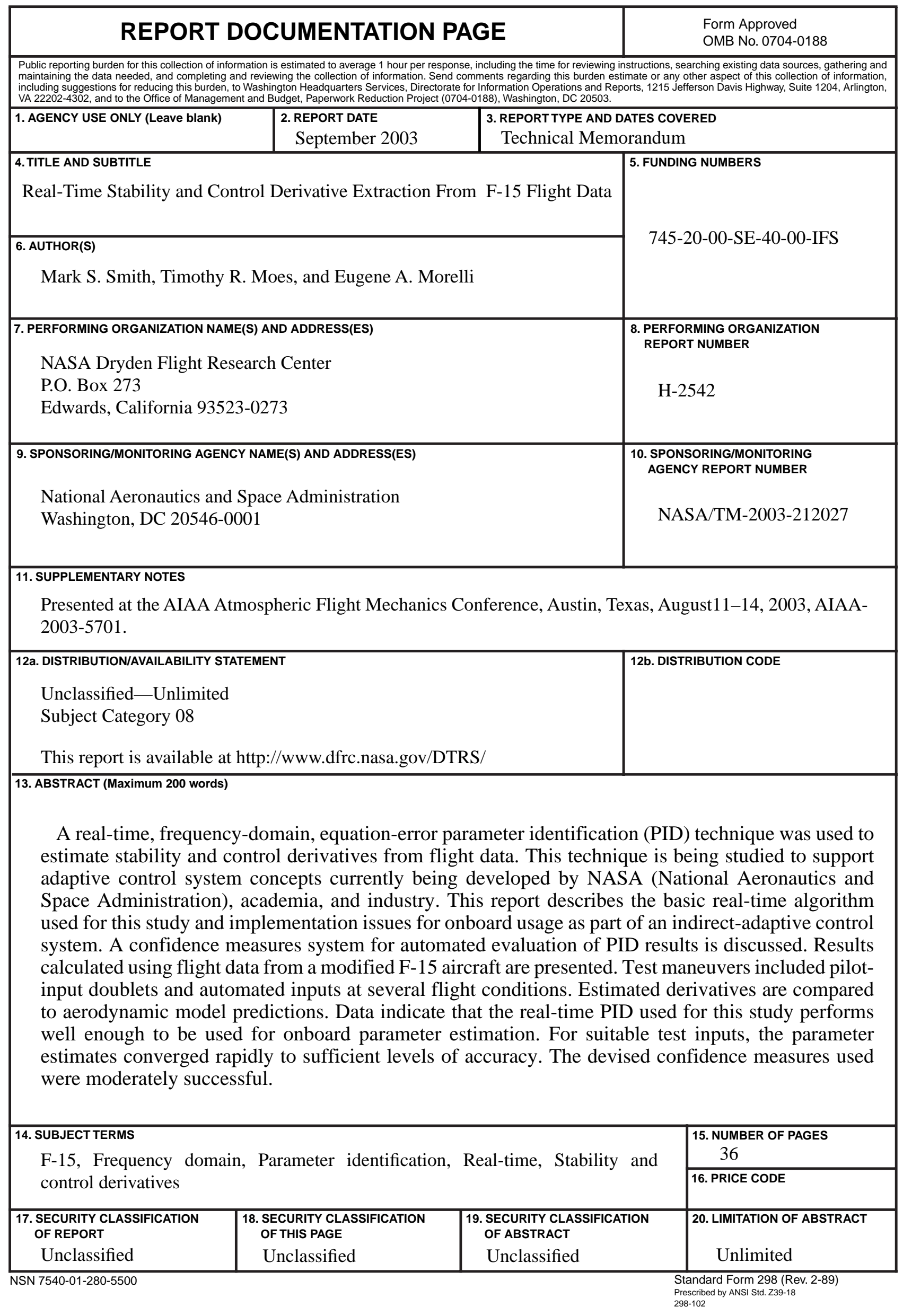

